\title{
Experimental Study of Low-Pressure Nitrogen Dielectric Barrier Discharge
}

\author{
Hasina Khatun* and A.K. Sharma ${ }^{\dagger}$ \\ Microwave Tubes Area, \\ Central Electronics Engineering Research Institute (CEERI) \\ (A constituent laboratory of Council of Scientific and Industrial Research, CSIR) \\ Pilani, Rajasthan-333031, INDIA \\ P.K. Barhai \\ Applied Physic Department, Birla Institute of Technology \\ Ranchi-835215, India \\ (Received on 27 September, 2010)
}

\begin{abstract}
The electrical and spectral characteristics of a dielectric barrier discharge (DBD) are experimentally investigated in a sealed off coaxial cylinder filled with nitrogen at a pressure of 10 mbar. The discharge is a transient diffused glow at low frequency alternating voltage $(60 \mathrm{~Hz})$ and changes to a filamentary mode at high frequency alternating voltage $(35 \mathrm{kHz})$. In case of pulsed voltage, the discharge is always transient diffused glow at any frequency. The intensity of a second positive system (SPS) of the nitrogen molecule has been also measured to characterize the discharge excitation. The effective vibrational temperature is estimated from the SPS vibrational band, $\Delta v=-2$. It is concluded that the intensity of the SPS of the nitrogen and the effective vibrational temperature depends upon the reduced electric field and the energy consumed per cycle by the device.
\end{abstract}

Keywords: Dielectric barrier discharge, Second positive system, effective vibrational temperature.

\section{INTRODUCTION}

The DBD based plasma sources are mercury free and environmental friendly. Therefore, the DBD technology is extensively adopted by many industries and scientific societies [12]. In the $\mathrm{DBD}$, the electrodes are coated with dielectric and the gas is filled in between the coated dielectric electrodes. The electrons gain electrical energy to initiate discharges by either alternating voltage or pulsed voltage. During the discharge, the gas is excited and the neutral gas temperature remains close to the ambient temperature. This property of plasma is called non-thermal non-equilibrium plasma.

The DBD sources are excited by low to high frequency operating voltage. Depending on the operating conditions, numerous investigations have been performed to understand and explain the physical basis of the discharge mode [2-6]. There are three types of discharge mode namely filamentary, diffused glow and glow. The DBD consists of microdischarges and randomly distributed over the gap [3]. These microdischarges are overlapped with each other and cover uniformly the entire dielectric surface, which in turn appear as the diffused glow and glow. In order to improve and implement the DBD technology for different type of applications, it requires studying the excitation processes in these discharge modes. The Townsend's theory helps to describe the low-pressure gas discharge. The detailed study of the sealed off coaxial low-pressure nitrogen DBDs will contribute the better understanding of the fundamental processes involved in DBDs. The DBD sources can exhibit non-thermal plasma property even at atmospheric pressure or above [6-7]. The atmospheric pressure conditions are most suitable for DBD based ultraviolet (UV)/ vacuum ultraviolet (VUV) excimer

\footnotetext{
*Electronic address: shamina \_seikh@yahoo.com

${ }^{\dagger}$ Electronic address: aks@ceeri.ernet. in

‡Electronic address: pkbarhai@bitmesra.ac.in
}

sources. In case of high-pressure discharge, the electron avalanche of critical size distorts the local electric field by the space charges of electrons and positive ions. This mechanism is called streamer and it is too tough to understand the streamer processes involved in high-pressure DBD.

This paper includes the electrical as well as the optical characteristics of the discharge mode at different excitation voltages. The experimental results of the low-pressure dielectric barrier discharge (LPDBD) source and also the extended studies of Wagnaars et al are described [2]. The diffused glow discharge mode appears at low frequency alternating voltage as well as unipolar-pulsed voltage excitation. The transient filamentary discharge is observed at high frequency alternating voltage. The discharge dynamics are also discussed in terms of the measured emission spectrum of nitrogen. The intensity of the emission spectrum describes the population of the vibrational states of the nitrogen molecular species. The effective vibrational temperature is calculated from the measured second positive system (SPS) of nitrogen molecule. The effective vibrational temperature affects the plasma reactivity. Further, the experimental results are used to analyse the discharge in terms of the energy consumed per cycle and the reduced electric field, $E / N$ where $E$ is the electric field and $N$ is the neutral gas density.

\section{EXPERIMENTAL ARRANGEMENT}

The sealed off cylindrical coaxial double barrier pyrex glass tube $\left(\varepsilon_{r}=5.6\right)$ of length $300 \mathrm{~mm}$ is fabricated. The gap is $3.25 \mathrm{~mm}$ filled with nitrogen $\left(N_{2}\right)$ gas at 10 mbar. The thickness of the pyrex glass is $2 \mathrm{~mm}$. The inner surface of the pyrex tube is covered by $0.05 \mathrm{~mm}$ thickness of solid CuSil alloy electrode which acts as powered electrode. And the outer surface is covered by copper mesh electrode, which is acting as ground electrode. The active discharge area is $61.23 \mathrm{~cm}^{2}$. The alternating voltage and unipolar-pulsed voltage of varying frequency are used to excite the low-pressure dielectric 


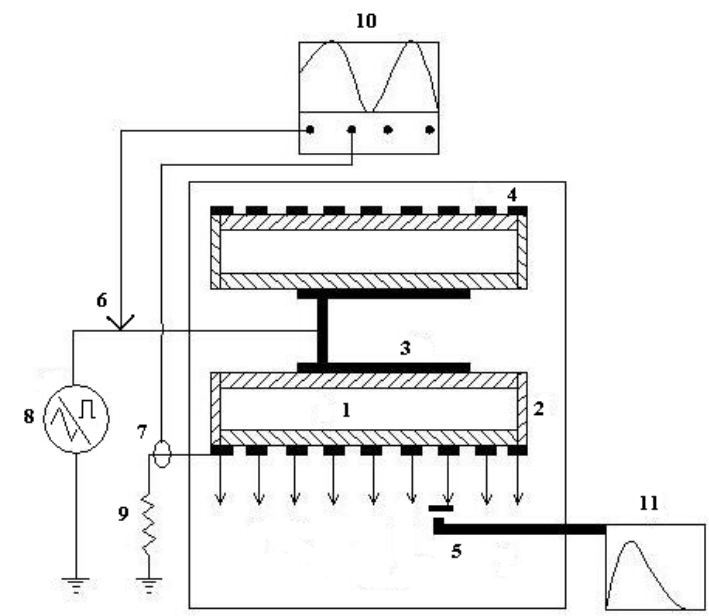

FIG. 1: Schematic diagram of experimental setup. (Gas gap-1, Pyrex-2, CuSil foil-3, Cu mesh-4, Collimated lens with optical fibre-5, High voltage probe-6, Current probe-7, Power supply-8, Resistance-9, Oscilloscope-10, Spectrometer-11)

barrier discharge (LPDBD) nitrogen. The high voltage probe (P6015A) and the current probe (Pearson) are used to measure the voltage waveforms across the DBD and the current waveforms through the DBD, respectively. The schematic of experimental setup is described in figure 1 . The digital oscilloscope (Tektronix TDS3034B, 500 MHz) synchronously displays the voltage and current signals. The characteristic spectrum of nitrogen is measured by a spectrometer (HR: 4000 , Ocean Optics) through an optical fiber and focused onto the slit of the spectrometer using a collimated lens.

\section{EXPERIMENTAL RESULTS}

\subsection{Electrical characteristics}

The discharge characteristics in terms of voltage and current waveforms at different operating conditions are shown in figures 2(a), (b), (c) and (d). At $60 \mathrm{~Hz}$ alternating voltage, the current waveform consists of large numbers of current pulses and the duration of each current pulse is more than $1 \mu \mathrm{s}$ (figure 2(a)). The current pulses occurs upto the maximum applied voltage during each half cycle. During the discharge, charges accumulate on the dielectric surface and the internal electric field builds opposite to the external alternating voltage. The discharge occurs in the form of the microdischarge and the extinction of these microdischarges takes place after few microseconds. Each microdischarge is suppressed by overlapping of the near by microdischarge feet and the transient diffusive glow discharge is appeared in the gas gap [8]. An increase in the driving frequency i.e. $35 \mathrm{kHz}$, the number of current pulses per half cycle decreases to few numbers and each current pulse of width few hundred nanoseconds (figure 2(b)). The amplitude of the current pulse at the high frequency alternating voltage is much higher than the low frequency alternating voltage. This implies that the number of electron density is higher at the high frequency alternating voltage [9].
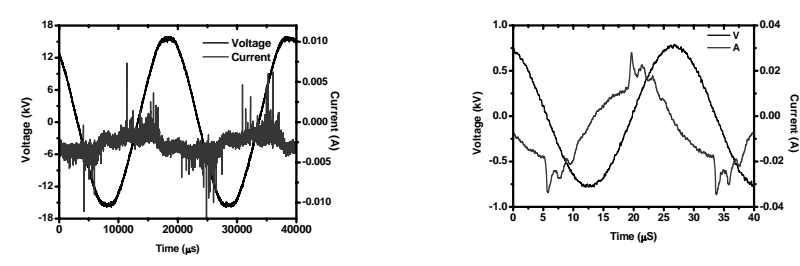

(a)

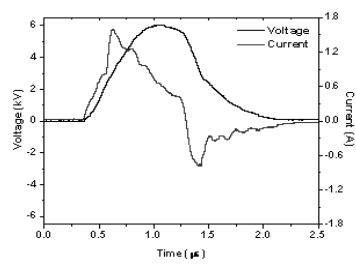

(b)

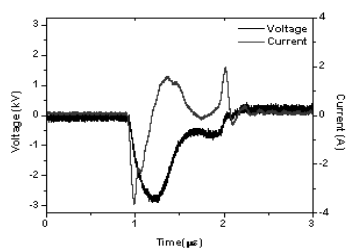

FIG. 2: I-V characteristics of nitrogen discharges excited by (a) 16 $\mathrm{kVp}, 60 \mathrm{~Hz}$ alternating voltage (b) $0.767 \mathrm{kV}_{p}, 35 \mathrm{kHz}$ alternating voltage (c) $6 \mathrm{kV}, 1 \mathrm{kHz}$ positive unipolar pulsed voltage and (d) 3 $\mathrm{kV}, 14 \mathrm{kHz}$ negative unipolar pulsed voltage (Pressure $=10 \mathrm{mbar}$ )

In case of nitrogen LPDBD driven by the unipolar-pulsed voltage, the current waveform is characterized by single peak at the rising and the trailing edge of the pulsed voltage irrespective of the applied voltage frequency (figures 2(c) and 2(d)). At the rising front of the voltage pulse, the primary discharge is occurred and the voltage falling flank is followed by the secondary discharge, without consuming the external applied energy. Thus, the discharge excitation by the unipolarpulsed voltage enhances the efficiency of the coaxial DBD source [9-11]. In figure 2(d), the sharp rise in current pulse is observed due to the small pulse width and fast rise time of the negative unipolar-pulsed voltage. The discharge current peak decreases slowly and covers the entire dielectric surface at the low applied unipolar-pulsed frequency $(1 \mathrm{kHz})$ and the pulse width is about $1 \mu \mathrm{s}$. The current waveform at low and high frequency, the unipolar-pulsed voltage confirms diffused type glow discharge [9]. The magnitude of the current pulses reaches few amperes unlike milliamperes in case of the alternating voltage excitation. This is due to the discharge current superimposed with the high displacement current.

\subsection{Optical characteristics}

The input electrical energy directly transfers to the electrons, results in electronic collision. The gaseous species are excited to the higher electronic states. These species decay from the higher electronic states, which emit photons of their characteristic wavelength. The emission spectrum of the SPS gives information of the molecular nitrogen species and its concentration under different discharge conditions.

Figure 3 shows that the measured emission spectrum consists of the molecular SPS band of nitrogen. According to the operating conditions, the occurrence of the SPS dominates over the first negative system (FNS) [12]. The population of the excited states $N_{2}\left(C^{3} \Pi_{u}\right)$ and $N_{2}^{+}\left(B^{2} \Sigma_{u}^{+}\right)$are caused by the direct electron impact excitation from the molecular 
Table 1 Electrical parameters and effective vibrational temperature at different operating condition

\begin{tabular}{ccccc}
\hline Applied voltage source & $\begin{array}{c}\text { Breakdown voltage } \\
(\mathrm{kV})\end{array}$ & $\begin{array}{c}\text { Reduced electric field } \\
\left(\mathrm{V} / \mathrm{m}^{2}\right)\end{array}$ & $\begin{array}{c}\text { Energy consumed per } \\
\text { cycle (mJ) }\end{array}$ & $\begin{array}{c}\text { Vibrational } \\
\text { temperature }(\mathrm{K})\end{array}$ \\
\hline $16 \mathrm{kV}_{\mathrm{p}}, 50 \mathrm{~Hz} \mathrm{AC}$ & 1.29 & $2.16 \times 10^{-16}$ & 5.7 & 14964 \\
\hline $1.56 \mathrm{kV}_{\mathrm{p}}, 35 \mathrm{kHz} \mathrm{AC}$ & 0.583 & $9.77 \times 10^{-17}$ & 0.18 & 10943 \\
\hline $6 \mathrm{kV}_{\mathrm{p}}, 1 \mathrm{kHz}, 1 \mu \mathrm{s}$ unipolar pulse & 2 & $3.352 \times 10^{-16}$ & 2 & 9338 \\
\hline $3 \mathrm{kV}_{\mathrm{p}}, 14 \mathrm{kHz}, 400$ ns unipolar pulse & 0.752 & $1.264 \times 10^{-16}$ & 9245 \\
\hline
\end{tabular}

ground state $N_{2}\left(X^{1} \Sigma_{g}^{+}\right)$or via the electron impact ionization of the nitrogen molecule $N_{2}^{+}\left(X^{2} \Sigma_{g}^{+}\right)$. The excitation energy of the states $N_{2}\left(C^{3} \Pi_{u}\right)$ and $N_{2}^{+}\left(B^{2} \Sigma_{u}^{+}\right)$for the corresponding vibrational level $v^{\prime}=0$, are $11.1 \mathrm{eV}$ and $18.7 \mathrm{eV}$, respectively. The transition from $N_{2}\left(C^{3} \Pi_{u}, v^{\prime}=0\right)$ to $N_{2}\left(B^{3} \Pi_{g}, v^{\prime \prime}=0\right)$ emits the characteristics photons of (0-0) band of the SPS at $337.1 \mathrm{~nm}$ [13]. Similarly, the subsequent radiative decays of the excited state $\mathrm{N} 2+(B 2 \Sigma+\mathrm{u}, \mathrm{v} /=0)$ to $N_{2}^{+}\left(X^{2} \Sigma_{g}^{+}, v^{\prime \prime}=0\right)$ emits the characteristics photons of (0-0) band of the FNS at $394.1 \mathrm{~nm}[13]$.

At low frequency alternating voltage, the nitrogen plasma shows only the SPS spectrum (figure 3 (a)). The high frequency alternating voltage and the unipolar pulsed excitation produces two to three times more intense peaks of the SPS band (figures 3 (b), (c), (d)). In these spectrum, few peaks of the FNS band are also observed (figures 3 (b), (c), (d)). The emission intensity of the particular wavelength from the excited state is proportional to the concentration of the species present in that excited state.

In LPDBD nitrogen, the excitations are also characterized in terms of the vibrational temperature, $T_{v}$. The vibrational states of second positive system, $\Delta v=-2$ helps to estimate $T_{v}$. For non-equilibrium plasma, the effective vibrational temperature is evaluated from the slope of $\log \left(I / N^{4}\right)$ against $G\left(v^{\prime}\right)$, where $I$ is the relative intensity and $G\left(v^{\prime}\right)$ is the vibrational term [14]. Figure 4 represents the graphs between $\log$ $\left(I / N^{4}\right)$ w. r. $t$ the term value.

\section{DISCUSSION}

The transient diffusive glow discharge is observed in the nitrogen LPDBD source driven by the low frequency alternating voltage and low to high frequency unipolar-pulsed voltage. During the discharge, the energy consumed per cycle is calculated using the breakdown voltage, applied frequency, applied peak voltage and capacitance of the double barrier dielectric discharge [15]. Table 1 shows the above listed parameters at different operating conditions. The breakdown voltage is high at the low frequency irrespective of the excitation voltage i.e. the alternating voltage and the unipolar pulsed voltage. In case of the unipolar-pulsed voltage, the rate of change of the applied voltage is fast in comparison to the alternating voltage, causes breakdown of the nitrogen gas at high voltage. The reduced electric field, $E / N$ at the high breakdown voltage is not sufficient enough to cause large excited species as shown in figure 3 (a). The value of $E / N$ is lower at $35 \mathrm{kHz}$ alternating voltage, which results into high intensity (figure 3 (b)). The fast rise time of the unipolarpulsed voltage increases the value of $E / N$, which results into

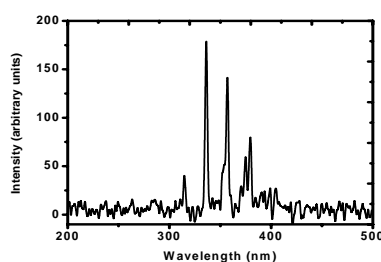

(a)

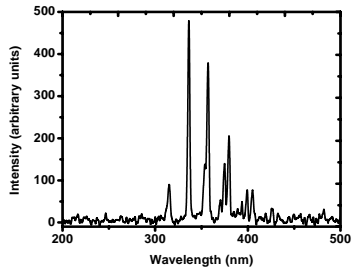

(c)

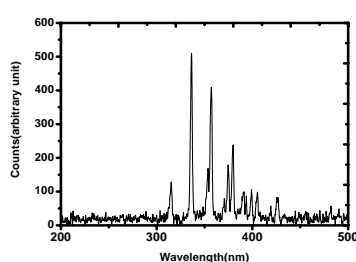

(b)

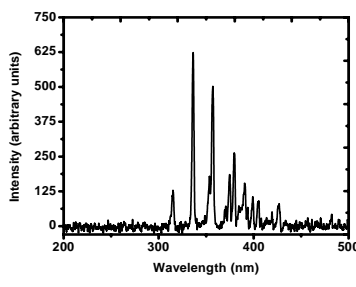

FIG. 3: Intensity spectrum of second positive system of nitrogen (10 mbar) observed in coaxial DBD driven by (a) $16 k V_{p}, 60 \mathrm{~Hz}$ alternating voltage, (b) $1.56 \mathrm{kV}, 35 \mathrm{kHz}$ alternating voltage, (c) 6 $\mathrm{kV}, 1 \mathrm{kHz}$ positive unipolar pulse (1 $\mu$ s pulse width) and (d) $3 \mathrm{kV}$, $14 \mathrm{kHz}$ negative unipolar pulse (400 ns pulse width).

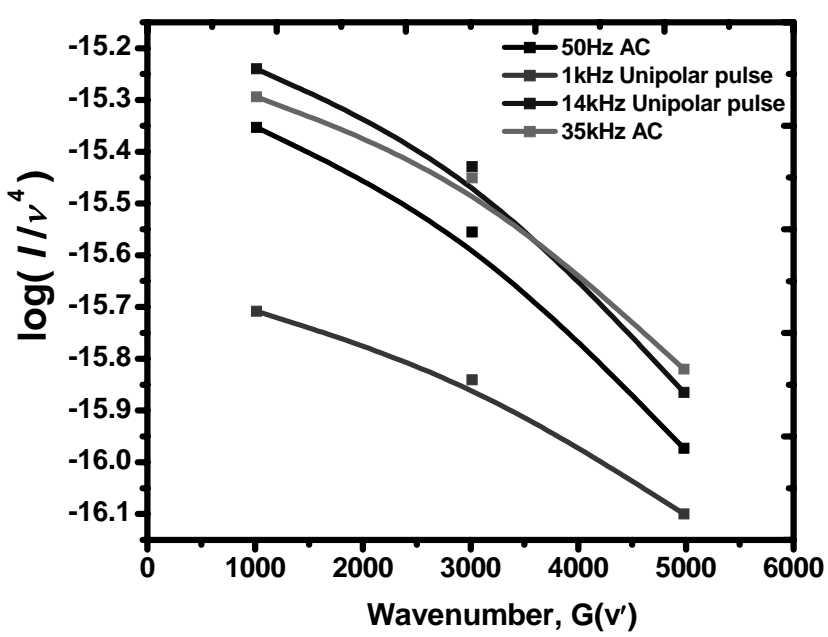

FIG. 4: Effective vibrational temperature of nitrogen in a LPDBD at 10 mbar pressure w. $r$. $t$ term value.

the high electron energy. The processes such as excitation, ionization and dissociation increase rapidly with increasing $E / N$. This results in increase of the plasma-chemical efficiency [16]. At the high frequency, the decay time of the radiation processes results in high intense peaks even the value 
of $E / N$ is low.

The measured emission intensity of (0-0) band of the FNS and SPS of nitrogen molecule shows that the dominating peak of the molecular band strongly depends upon the input electrical power and also on the operating conditions. The effective vibrational temperature is calculated from the slope of $\log \left(I / N^{4}\right)$ against $\mathrm{G}(\mathrm{v})$ from figure 4 and is listed in Table 1 . The effective vibrational temperature is low when excited by unipolar-pulsed voltage in comparison to alternating voltage. From the above statement, we can conclude that the heat generation will be less in case of unipolar-pulsed voltage excitation. The effective vibrational energy also decreases with decrease in the energy consumed per cycle by the DBD source. The results of effective vibrational energy are also in good agreement with well-known correlation [12-13].

The experimental results at the low pressure follow the discharge characteristics operating at high pressure [17]. Either alternating voltage or pulsed voltage excitation, initially the low-pressure discharge follows the Townsend breakdown mechanism and later formation of microdischarges is observed. According to Wagnaars et al, the low-pressure argon DBD consists of glow discharge like dc glow discharge2. The results presented in this paper show the transient diffusive glow discharge as well as filamentary discharge. This is due to the high operating pressure, smaller gas gap and dif- ferent excitation method in comparision to Wagnaars et al.

\section{CONCLUSION}

The microdischarges occur in the low-pressure nitrogen DBD and overlapped by nearby microdischarge feet to cause diffused glow like discharge in case of low frequency alternating voltage and low to high frequency unipolar pulsed voltage. The electrical and spectral behaviour is discussed in terms of the reduced electric field and the effective vibrational temperature, respectively. The pulsed voltage with the fast rise time and the high frequency shows the maximum emission intensity at high reduced electric field. The emission spectrum of the nitrogen mainly consists of SPS band and the vibrational temperature depends upon the energy consumed per cycle as well as reduced electric field.

\section{Acknowledgement}

Authors acknowledge the support and encouragement of Director, CEERI Pilani and Dr. SN Joshi, Emeritus Scientist, CEERI Pilani.
[1] U. Kogelschatz, Plasma Chemistry and Plasma Processing 23 (2003) 1.

[2] E. Wagnaars, Plasma Breakdown of Low-Pressure Gas Discharges, Ph. D Thesis Technische Universiteit Eindhoven, 2006.

[3] N. Gherardi, G. Gouda, E. Gat, A. Ricard and F. Massines, Plasma Sources Sci. Technol. 9 (2000) 340.

[4] Hasina Khatun, Alok Mishra, Mahesh Kumar, U.N. Pal, A.K. Sharma and P.K. Barhai, Indian Journal of Pure \& Applied Phy. 46 (2008) 889.

[5] U.N. Pal, A.K.Sharma, J.S.Soni, Sonu Kr, H. Khatun, M. Kumar, B.L.Meena, M.S. Tyagi, B-J Lee, M. Iberler, J Jacoby and K. Frank, J. of Phys D: Appl. Phys 42 (2009) 45213.

[6] U. Kogelschatz, IEEE Trans. On Plasma Sci. 30 (2002) 1400.

[7] U. Kogelschatz, Plasma Sources Sci. Technol. 11 (2002) A1.

[8] J. Rahel, M. SAra, P. Stahel and D. Trunec, Contrib. Plasma Phys. 47 (2007) 34.

[9] R.P. Mildren and R.J. Carman, J. Phys. D: Appl. Phys. 34 (2001) L1.

[10] S. Liu, Electrical modeling and unipolar-pulsed energization of dielectric barrier discharge: Ph.D Thesis Electronics and Information Techniques (University of Karshuruhe), Germany (2002).

[11] R.J.Carman and R.P.Mildren, J. Phys. D: Appl. Phys. 36 (2003) 19.

[12] F. Iza and J.A. Hopwood, IEEE Trans. On Plasma Sci. 32 (2004) 498.

[13] D.C.Tytet, Proc. Phys. Soc. 80 (1962) 560.

[14] G Herzberg, Molecular Spectra and Molecular Structure: I: Spectra of Diatomic Molecules, D. Van Nostrand company, INC., Princeton, New Jersey, New York, 1950.

[15] U. Kogelschatz, B Eliasson, Ozone generation and applications, Handbook of Electrostatic Processes (J S Chang, A. J. Kelly, J. M. Crowley, eds), p.581. Marcel Dekker, New York (1995).

[16] P. Bletzinger and B.N. Ganguly, J. Phys. D: Appl. Phys.36 (2003) 1550.

[17] J Shin and L L Raja, J Appl. Phys. 94 (2003) 7408. 